\title{
Prevalence of findings compatible with carotid artery calcifications on dental panoramic radiographs
}

\author{
Stefan Bayer • Ernst-Heinrich Helfgen • Carolin Bös • \\ Dominik Kraus • Norbert Enkling • Sebastian Mues
}

Received: 22 October 2009 /Accepted: 19 April 2010 /Published online: 30 April 2010

(C) Springer-Verlag 2010

\begin{abstract}
Cerebrovascular accidents are responsible for killing or disabling more than half a million Americans every year. They are the third leading cause of death in this country. In Germany, the annual stroke incidence reaches 182 cases per 100,000 inhabitants. Stroke there is the fourth leading cause of death. There is a need of finding cost-effective means of decreasing stroke mortality and morbidity. Instruments for early diagnosis are of great humanitarian and economic importance. All possible clinical findings should be taken into account. It is not the demand of this study to present the panoramic radiograph as a screening test method for early diagnosis of atherosclerosis. The aim is to show the potential of this radiograph used in everyday clinical dental practice by the prevalence of radiopaque findings in the carotid region. This study included panoramic dental radiographs of 2,557 patients older than 30 years of age. Fifty-nine percent of the patients were women and $41 \%$ were men. The radiographs were adjudged for signs compatible with carotid arterial calcifications appearing as a radiopaque nodular mass adjacent to the cervical vertebrae at or below the intervertebral space C3-4. Of all these radiographs, $4.8 \%$ showed radiopaque findings compatible with atherosclerotic lesions. The proportion of women reached $64.8 \%$ and that of men reached $35.2 \%$. In accordance to recent literature, the results of this study show that about $5 \%$ of the patients show radiological findings
\end{abstract}

\footnotetext{
S. Bayer • E.-H. Helfgen · C. Bös · D. Kraus • N. Enkling •

S. Mues

Department of Prosthodontics,

Preclinical Education and Dental Materials Science, University of Bonn,

Bonn, Germany

S. Bayer $(\bowtie) \cdot N$. Enkling

Clinic for Prosthetic Dentistry, University of Bern,

Bern, Switzerland

e-mail: sbayer@uni-bonn.de
}

compatible with carotid arterial calcifications. Some of these patients at risk for a cerebrovascular accident may be identified in the dentist's office by appropriate review of the panoramic dental radiograph. The suspicion of carotid artery calcifications demands an impetuous referral to an appropriate practitioner who can assist in the control of risk factors and if necessary arrange surgical removal of the carotid arterial plaque. So, the dentist should be aware of this problem and able to make a contribution to stroke prevention.

Keywords Dental panoramic radiograph - Atherosclerosis . Stroke $\cdot$ Stroke prevention $\cdot$ Cardiovascular accident

\section{Introduction}

The account of screening tests and early diagnosis concepts attaches great importance in medical diagnostics. The possibility is offered to take advantage of early intervention and thus of a decrease in mortality and morbidity.

Cardiovascular disease, resulting in stroke or heart attack, is still the leading cause of death of men and women in Germany. Often, they cause an early death below 70 years and thus reduce the life span far below the mean age [1]. In the USA, for example, there were 4.6 million people in 2002 who had to live impaired by the physical and psychological effects of stroke and 12.6 million affected by the effects of heart attack [2].

The arteria carotis communis shows a bifurcation at the carotid bulb. This is where the arteria carotis externa and arteria carotis interna originate. Predilection spots for atherosclerotic lesion are often located at such anatomic structures due to hemodynamic effects of turbulences at the streaming blood, which increase the risk of plaque and intima erosion [3, 4]. 
Diagnostic inspection of carotid arterial calcifications is often done by using ultrasonic exploration $[5,6]$. This enables the examiner to get information about the vascular morphology and hemodynamic parameters. Also, the grade and length of the stenosis can be measured. The advantage of ultrasonic techniques is the absence of X-rays, which always have to be seen as a risk factor. In addition to ultrasonic exploration, catheter-based cerebral angiography is the gold standard of diagnostics in angiographic exploration of vascular diseases [7].

All these techniques as well as the panoramic radiograph show a more or less invasive treatment protocol combined with advantages and disadvantages. Contrast agents are administered intravascularly, X-rays are used for imaging, and complex manual procedures are needed which are individually performed with more or less quality depending on the examiner's experience [8-10]. Although the panoramic radiograph is not as complicated as a catheterbased cerebral angiography, it is necessary to be informed about its specific recommendations and to be exercised in the treatment protocol to be able to provide the best radiographic services possible to patients [11].

Methods which are cheap, of low risk, and comprehensive for early diagnosis of atherosclerotic disease are needed. The adjudgement of standard dental panoramic radiographs can be used as support to normal screening techniques. For the examiner as well as for the patient, there is approximately no further effort because this type of radiograph is used often in dental practice when the dental status is taken with new patients. Also as a diagnostic method for clarification of several questions, this radiograph is a part of everyday clinical practice $[12,13]$.

The dental panoramic radiograph is the only radiological technique, which does not only show the dental structures. Also, the jaws and facial structures are presented as well as the adjacent structures of the face and neck. Thus, the critical region of the carotid artery is brought out and this radiograph is able to show clinical findings of primary interest. The dentists are the only medical specialists to produce a great amount of radiographs with low radiation exposure. The effective radiation dosage reaches about 0.005 to $0.03 \mathrm{mSv}[14,15]$ and is far beyond the doses of a computer tomographyassisted angiography with about 2 to $4 \mathrm{mSv}$ [16].

In the past, some studies were made to see how often calcified plaques can be found in the standard patient population of the dental practice. In 1981, Friedlander et al. showed in a retrospective study that about $2 \%$ of 1,000 male veteran patients showed such signs of atherosclerosis [17]. Later, they showed that in a cohort of 19 patients suffering stroke caused by plaque embolization of calcified carotid artery, $37 \%$ showed findings compatible with plaques in the panoramic radiograph [18]. Demographically speaking, this is equivalent to $2 \%$ of the whole population. Carter et al. showed a prevalence of $3.6 \%$ findings compatible with calcifications in the carotid artery with 1,175 patients [19]. Additionally, the patients' anamnesis was enquired for further risk factors of a cardiovascular disease. A correlation of overweight and calcified plaques was found. Other risk factors did not show such a good correlation. Whether the knowledge of existing risk factors is correlated with a rise of calcified lesions in the dental panoramic radiograph was examined by Friedlander und Maeder [20]. They examined radiographs of patients with insulin-dependent diabetes mellitus and a control group without such a risk. The diabetics showed findings compatible with calcified plaques in $20.4 \%$ whereas the control group reached only $4 \%$. Up to now, the biggest study was performed with a study population of 1,879 patients by Cohen et al. [21]. They adjudged radiographs for the period from 1986 to 2000 . Only men 55 years of age or more who showed no neurological symptoms as sign of a cerebrovascular insult were included. Cardiovascular risk factors were identified in all patients through their anamnesis; $3.8 \%$ showed findings compatible with calcified lesions in the panoramic radiograph and $85.9 \%$ of these patients had at least one risk factor for cardiovascular problems to be found in the anamnesis. In $73 \%$, there were two or more risk factors found. The combination of calcified lesions and risk factors allows an impetuous referral to an appropriate practitioner who can assist in the control of risk factors and if necessary arrange surgical removal of the carotid arterial plaque. This offers the chance of stroke or heart attack preventive therapy. Craven et al. [22] were able to show the correlation between carotid artery and coronary artery stenosis. They examined 343 patients with and 167 patients without atherosclerotic heart disease by ultrasonic examination and showed the possibility of using carotid stenosis as an indicator for such heart disease.

Aim of the study

The aim of the study is a retrospective evaluation of dental panoramic radiographs due to the prevalence of radiopacities compatible with calcified atherosclerotic plaque of the carotid artery. The value of this radiograph for early diagnosis of cardiovascular problems should be described to sensibilize the dentist for the potential of this technique for stroke prevention.

\section{Materials and methods}

\section{Patient population}

Dental panoramic radiographs of all patients treated in the Center of Dento-Maxillo-Facial Medicine at the University of Bonn from 2000 to 2008 were included. Of all these radiographs, 2,575 fulfilled the inclusion criteria and were adjudged for signs compatible with calcified atherosclerotic plaque at the carotid artery. 
The radiographs of this period were established by using an Orthophos Plus 3 (Fa. Siemens, Bensheim, Germany). The appraisal was performed with a standardized illumination box (Modell DSK $18 \times 30$, Fa. Maier, GarmischPartenkirchen, Germany). The radiographs were examined three times and magnified by using a magnifying glass.

\section{Inclusion criteria}

The patients were selected as they had to be older than 30 years up to the time of the preparation of the radiograph. Compared to the study of Cohen et al. [21] who chose only male subjects for their study with 1,879 patients, the study presented was not influenced by the gender of the respondents. Although of great importance for clinical diagnostics and obligatory for every patient treatment, the case history was not referred to for this retrospective study. The aim was only to show the prevalence of the described radiological findings in the panoramic radiograph of the described cohort.

The findings compatible with carotid arterial calcification structures were defined appearing as an irregular, heterogeneous, or verticolinear nodular mass adjacent to the cervical vertebrae at or below the intervertebral space C3-4 and inferior to the angle of the mandible [23, 24]. Figure 1 gives an idea of the standard localization.

As differential diagnosis of these arterial calcifications, calcified triticeus cartilage, superior cornu of calcified thyroid cartilage, greater cornu of hyoid bone, sialoliths, and calcified lymph nodes can occur. Most of them appear in the cranial or caudal position of the carotid arterial calcification position [23, 24].

\section{Study setup}

An especially trained medical doctor adjudged the radiographs. This adjudgement was performed by using the illumination box and the magnifying glass at standardized conditions in a darkened room. The search aimed for radiopaque areas in the region of the carotid vessels. The search was performed as qualitative analysis. Figures 2, 3, 4, and 5 show examples of radiographs and the findings which were assessed to be relevant structures compatible with atherosclerotic lesions. The documentation included the age of the individual when the radiograph was taken and the sex of the individual.

\section{Results}

The study adjudged retrospectively 2,557 dental panoramic radiographs of patients older than 30 years. Fifty-nine percent of the study population showing positive radiographs were female and $41 \%$ were male individuals. Adjudged as positive due to the appearance of findings compatible with carotid artery calcification, there were 125 radiographs found. This means that $4.8 \%$ of all the radiographs adjudged at this cohort showed signs compatible with carotid artery calcification.

Of the 125 patients with such findings, there were 81 $(64.8 \%)$ of female and $44(35.2 \%)$ of male sex. The median age was 64.6 years when the radiograph was made. The youngest patient was 36 years old and of male sex. The oldest was 88 years old and of female sex. Figure 6 shows the distribution of age.

\section{Discussion}

Comparison to recent literature

The prevalence of signs compatible with calcified plaque at the carotid artery of dentists' patients is stated to be at about $3-5 \%$ in different patient groups by the recent literature [18,
Fig. 1 Diagram of a dental panoramic radiograph showing the main anatomic structures: $A$ maxillary sinus, $B$ nasal cavity, $C$ maxilla, $D$ mandible, $E$ vertebral body, $F$ hyoid bone, $G$ relevant region for the appearance of radiopaque shadow interpreted as atherosclerotic lesions of the carotid artery

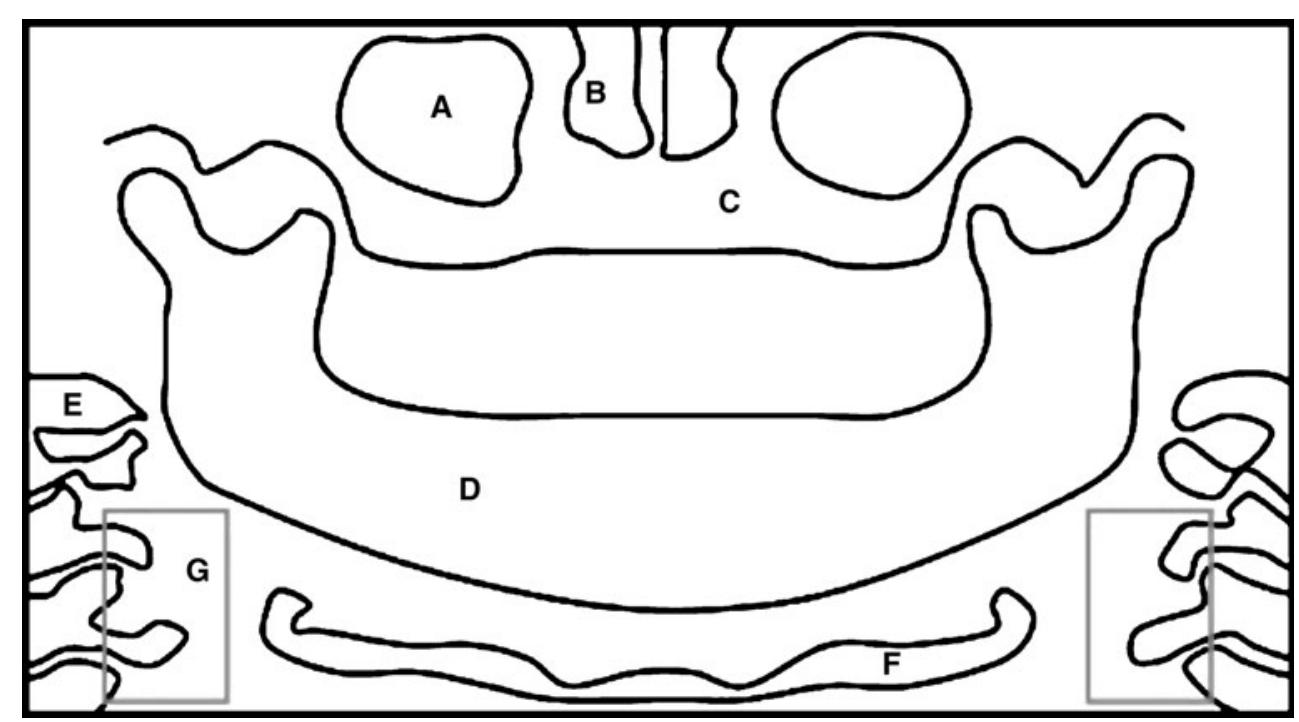




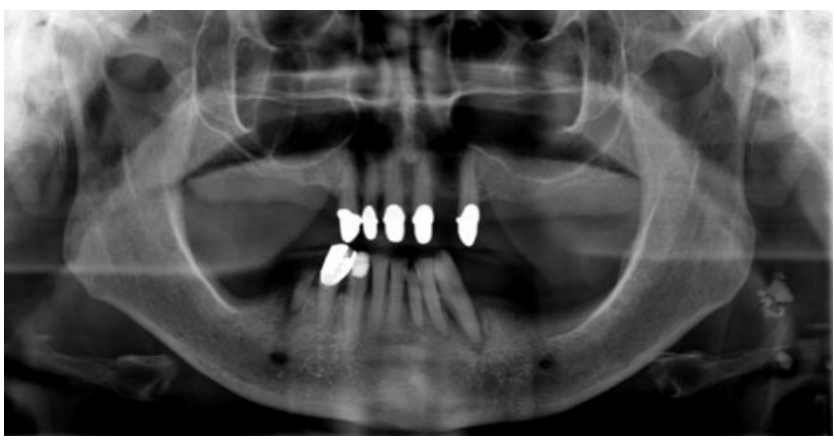

Fig. 2 Dental panoramic radiograph of a 72-year-old male patient with different dental findings and a radiopaque nodular mass at the left side adjacent to the cervical vertebrae at intervertebral space C3-4 in terms of an atherosclerotic lesion of the carotid artery. The marked region is shown in detail in Fig. 3

20, 25-28]. This value is in good accordance to the results of the study presented here as it examined a patient cohort only selected by their age.

The study design is a little different in all the studies. In the study presented here, the patient population was only determined by the age of 30 years or more. There was no selection done by sex or cardiovascular risk factors. Thus, such a high number of patients could be included, as it was not found in recent literature before.

The study wanted to show the prevalence in a mixed cohort of middle-aged and older patients. Some other studies did also refer to a cohort of patients about 30 years old and older [29]. The study of Tohno and Tohno showed that manifestation of calcifications is age-related and rising significantly by the patient's age [30]. It is clear that in younger patient cohorts only a minor number of cases with carotid calcifications can be found. Nevertheless, the accumulation of calcium in the aorta starts to occur after the age of 20 years [31-33]. The grade of atherosclerosis is higher in the aorta and coronary artery than in the cerebral

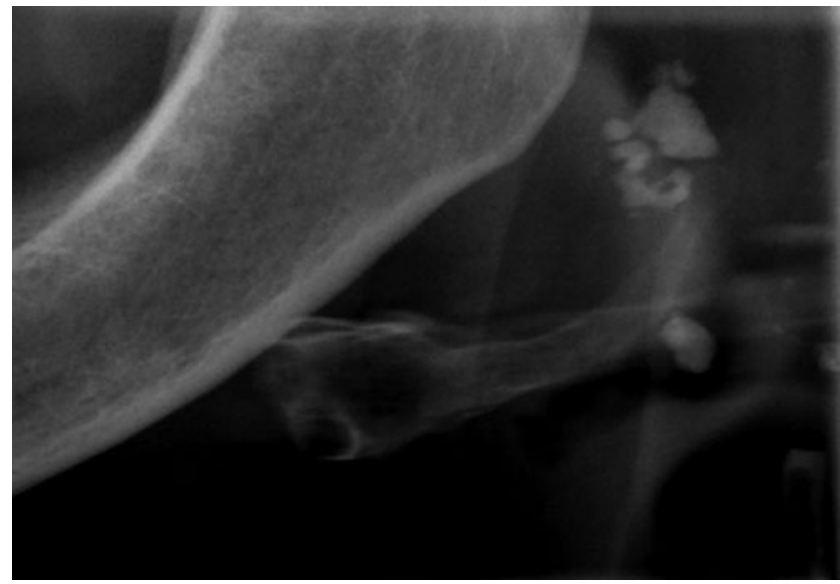

Fig. 3 Detail of the nodular mass adjacent to the cervical vertebrae at intervertebral space C3-4 shown in Fig. 2 at the right side of the radiograph

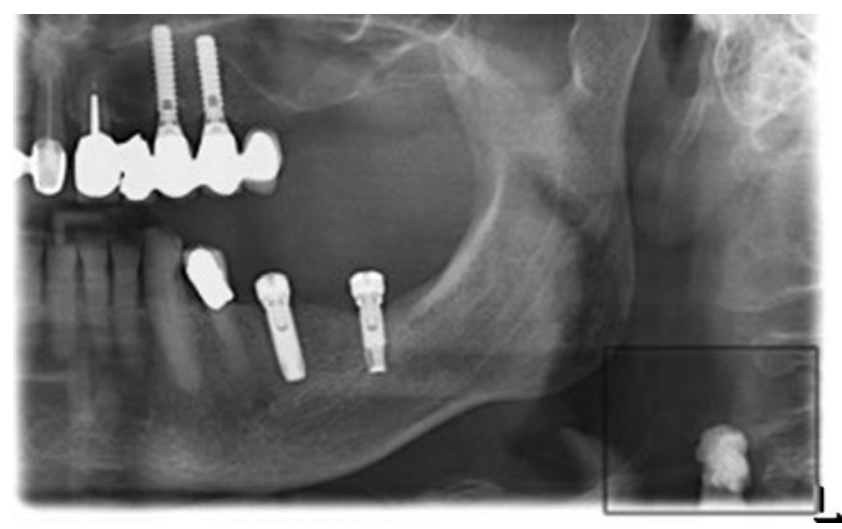

Fig. 4 Panoramic radiograph of a 67-year-old male patient showing a nodular mass adjacent to the cervical vertebrae at intervertebral space C3-4 besides multiple dental findings. The marked region is shown as detail in Fig. 5

arteries [34]. Despite these facts, the cohort also included patients of only about 30 years of age to be sure of adjudging almost all age groups possibly showing signs of calcified carotid arteries.

Much higher prevalence than in the study presented was found by Friedlander and Maeder [20] examining the prevalence of findings compatible with calcified carotid atherosclerosis in a cohort of 49 patients with insulindependent diabetes and older than 55 years of age. The prevalence in this cohort reached, as expected, $20.4 \%$. This much higher prevalence shows the importance of anamnesis for risk prediction.

As expected, a cohort of patients affected by a cardiovascular event like stroke or transient ischemic attack showed a high rise in signs compatible with calcified carotid atherosclerosis. Friedlander et al. [18] examined 19

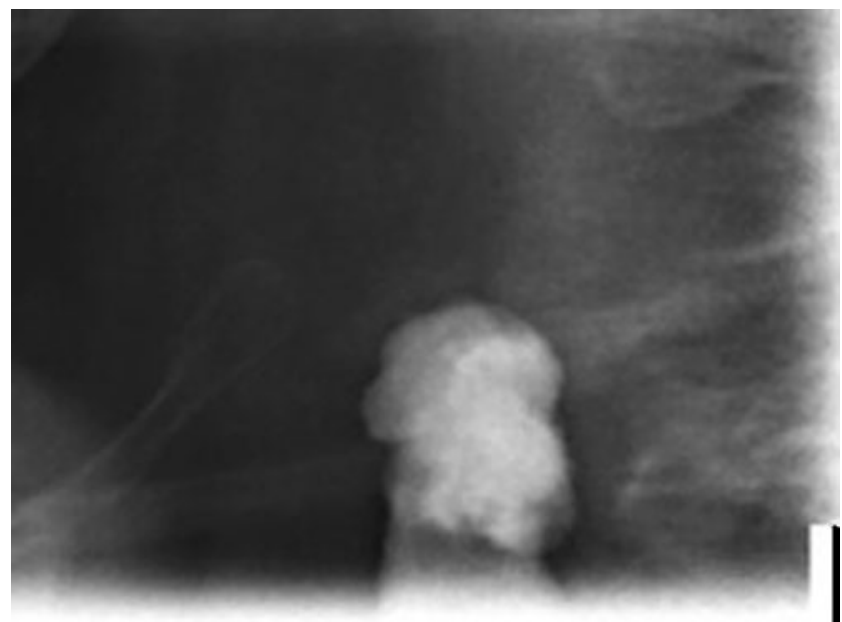

Fig. 5 Detail of the nodular mass adjacent to the cervical vertebrae at intervertebral space C3-4 shown in Fig. 3 at the left side of the radiograph. The structure can be interpreted as a calcified atherosclerotic lesion of the left carotid artery 


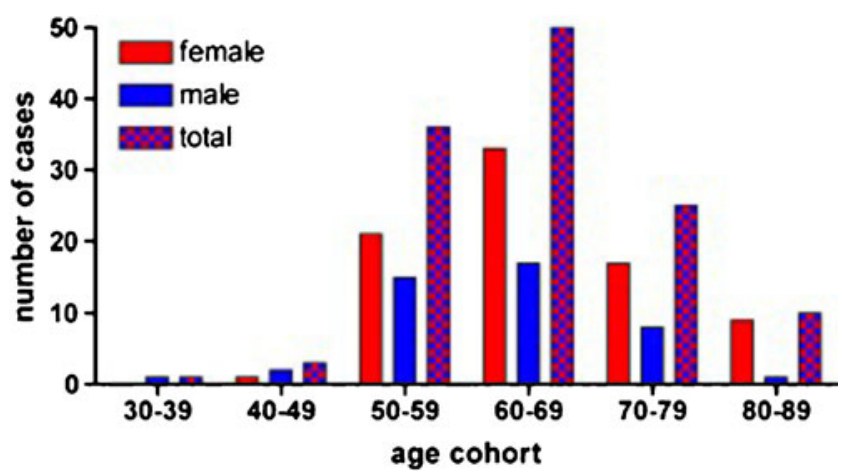

Fig. 6 Distribution of age of the cohort differentiated by female and male sex and for the whole cohort. Eighty-one (64.8\%) of the 125 patients were women and $44(35.2 \%)$ were men. The median age reached 64.6 years. The youngest patient was of male gender and 36 years old, the oldest one was 88 years and of female gender

patients of such anamnesis and found calcified atherosclerotic lesions with $37 \%$ of them.

If the data of the prevalence are combined with the study of Aronow et al., the importance of the panoramic radiograph for stroke prevention is underlined. Aronow found in a prospective study that older patients with massive signs of carotid artery atherosclerosis have a 2.5 times higher risk of atherothrombotic brain infarction [35].

The problem of the connection between radiographical identifiable atherosclerotic lesions and the grade of carotid stenosis was examined by Madden et al. [29]. Fifty-two patients received a duplex sonography as gold standard for stenosis examination. All of the arteries showed signs of stenosis of different grading. Afterwards, panoramic radiographs were made and adjudged by two examiners. Patients with low-grade stenosis in the sonography results showed radiopaque lesions of the carotids in $25-31 \%$ and higher grades of stenosis only in $21-23 \%$ of the cases. This shows the necessity of further examination of the carotids when the panoramic radiograph shows radiopaque lesions in the carotid region.

The dental panoramic radiograph does not have the potential of the duplex sonography for early diagnosis and stroke prevention, but it can be an additional valuable method. As it does not require any additional time or cost, its information concerning the carotid vessel should be used as a coproduct of a standard dental procedure.

\section{Differential diagnosis and localization}

There are different anatomic and pathologic radiopacities in close proximity of the region where findings compatible with carotid calcifications can be found in panoramic radiographs. In addition to that fact, a complicating factor in relation to the differential diagnosis is the range of age. This range shows also the greatest prevalence for findings compatible with atherosclerotic signs in the carotid artery and coincides with the greatest occurrence of mineralization and/or calcifications that are reported as being responsible for false diagnoses, for example, ossification of the triticeous, cricoid, and thyroid cartilages [19, 23, 36]. Calcifications caused by carotid atherosclerotic plaque are used to be found as radiopacities inferior to the angle of the mandible and the hyoid bone, superior to the thyroid cartilage adjacent to the cervical vertebrae at or below intervertebral space C3-4 [23, 24]. Further possible pathologic radiopacities are calcified lymph nodes, phleboliths, calcifications of the submandibular gland, or sialoliths [23].

Criticism of the method

A positive aspect of this study is the huge patient population. The panoramic radiograph is a standard instrument of dental diagnostics for new patients and after restoration of dentition in recall concepts [12, 13, 15, 37]. So, this radiograph can be seen as a chance and an addition for the diagnosis of macroangiopathy.

At the Center of Dento-Maxillo-Facial Medicine at the University of Bonn, about 2,000 dental panoramic radiographs are performed per year. The personnel are trained to get a standardized technique of radiograph, which is also possible in any dental practice. The dose of radiation is very low. The method is cheap in production and archival storage. Although these are advantages in comparison with the standard diagnostic methods used for carotid calcifications, the lower diagnostic output has to be mentioned as a problem. The panoramic radiograph cannot be recommended as a screening method to be used in general. The findings in carotid calcification cannot yet be correlated with the grade of stenosis [38]. In addition, the risk of erroneous diagnosis by the multiple differential diagnosis mentioned above is a problem and increases by summation effects of structures at the two-dimensional radiograph. Whether the radiopacities found in this study were clinically relevant for blood flow and stroke risk was not evaluated. In addition to that question, it is not possible to detect plaques which are not calcified by this radiological technique.

Another weak point of this radiograph was found at the adjudgement. Sometimes the markers, which include patient data and the orientation of the sides by a capitalized $\mathrm{L}$, were positioned in a way covering the region of possible signs compatible with carotid calcification findings that make it impossible to accomplish correct and good adjudgement of the critical region. These markers were placed without knowledge of the problem of such findings in this radiograph. The practitioner should be aware of this problem to be able to choose another marker position. 


\section{Conclusion}

The presence of atherosclerotic carotid calcification is a risk factor for the appearance of cardiovascular problems [18-20, 22, 39]. The study by Madden et al. [29] tries to show that the dental panoramic radiograph shows low sensitivity and positive predictive value in positive findings suspicious to be carotid calcification. The panoramic radiograph cannot be considered an accurate test for stenosis, but the positive finding in a radiograph requires referral to a specialist for further diagnostics as it cannot be negated that there is a connection between findings in the panoramic radiograph and cardiovascular events like stroke. Thus, the dentists are the only specialists to see a huge population by being able to use a radiograph for stroke and heart attack prevention without the need of further radiation dose, costs, and time-consuming procedures. The technique of this radiological method allows radiographs of good reproducibility, and the diagnostic with this radiograph needs only little special information and skills to reach good results. It is a fact that this method is not suitable as a single component of diagnostics or as a standard screening method. An exact estimation of the risk is also not possible $[29,40]$. Nevertheless, the diagnostic information reached by this method makes it worth using as an amendment of the standard procedures, especially because it is a cost and risk-free extra information. It is possible to use this radiograph as an important component of early diagnosis in atherosclerosis [20, 40-44]. So, the dentist can indeed help save lives by careful evaluation of the panoramic radiograph.

Conflict of interest statement The authors declare that they have no conflict of interest.

\section{References}

1. Robert-Koch-Institut (2007) Health in Germany. Health Report of the Federal Department

2. American Heart Association (2002) Heart and stroke statistical update. American Heart Association, Dallas

3. Böcker W, Denk H, Heitz PU (1996) Pathologie. Urban \& Schwarzenberg, München, pp 227-235

4. Ogata J, Masuda J, Yutani C, Yamaguchi T (1990) Rupture of atheromatous plaque as a cause of thrombotic occlusion of stenotic internal carotid artery. Stroke 21:1740-1745

5. Muller M, Ciccotti P, Reiche W, Hagen T (2001) Comparison of color-flow Doppler scanning, power Doppler scanning, and frequency shift for assessment of carotid artery stenosis. J Vasc Surg 34:1090-1095

6. Jaff MR, Goldmakher GV, Lev MH, Romero JM (2008) Imaging of the carotid arteries: the role of duplex ultrasonography, magnetic resonance arteriography, and computerized tomographic arteriography. Vasc Med 13:281-292

7. Latchaw RE, Alberts MJ, Lev MH, Connors JJ, Harbaugh RE, Higashida RT, Hobson R, Kidwell CS, Koroshetz WJ, Mathews
V, Villablanca P, Warach S, Walters B (2009) Recommendations for imaging of acute ischemic stroke: a scientific statement from the American Heart Association. Stroke 40:3646-3678, A journal of cerebral circulation

8. Kauffmann GW, Moser E, Sauer R (2001) Radiologie. Urban \& Fischer, München, pp 91-96

9. White SC, Heslop EW, Hollender LG, Mosier KM, Ruprecht A, Shrout MK (2001) Parameters of radiologic care: an official report of the American Academy of Oral and Maxillofacial Radiology. Oral Surg Oral Med Oral Pathol Oral Radiol Endod 91:498-511

10. Jahromi AS, Cina CS, Liu Y, Clase CM (2005) Sensitivity and specificity of color duplex ultrasound measurement in the estimation of internal carotid artery stenosis: a systematic review and meta-analysis. J Vasc Surg 41:962-972

11. Goren AD, Lundeen RC, Deahl ST 2nd, Hashimoto K, Kapa SF, Katz JO, Ludlow JB, Platin E, Van Der Stelt PF, Wolfgang L (2000) Updated quality assurance self-assessment exercise in intraoral and panoramic radiography. American Academy of Oral and Maxillofacial Radiology, Radiology Practice Committee. Oral Surg Oral Med Oral Pathol Oral Radiol Endod 89:369-374

12. American Dental Association Council on Scientific Affairs (2006) The use of dental radiographs: update and recommendations. J Am Dent Assoc 137:1304-1312

13. American Dental Association (2004) The selection of patients for dental radiographic examinations. American Dental Association, Chicago

14. Ngan DC, Kharbanda OP, Geenty JP, Darendeliler MA (2003) Comparison of radiation levels from computed tomography and conventional dental radiographs. Aust Orthod J 19:67-75

15. Rother U (2001) Modern imaging diagnostics in dentistry (Moderne bildgebende Diagnostik in der Zahn-, Mund- und Kieferheilkunde). Urban \& Fischer, München, pp 52-65

16. Bor D, Toklu T, Olgar T et al (2006) Variations of patient doses in interventional examinations at different angiographic units. Cardiovasc Intervent Radiol 29:797-806

17. Friedlander AH, Lande A (1981) Panoramic radiographic identification of carotid arterial plaques. Oral Surg Oral Med Oral Pathol 52:102-104

18. Friedlander AH, Manesh F, Wasterlain CG (1994) Prevalence of detectable carotid artery calcifications on panoramic radiographs of recent stroke victims. Oral Surg Oral Med Oral Pathol 77:669673

19. Carter LC, Haller AD, Nadarajah V, Calamel AD, Aguirre A (1997) Use of panoramic radiography among an ambulatory dental population to detect patients at risk of stroke. J Am Dent Assoc 128:977-984

20. Friedlander AH, Maeder LA (2000) The prevalence of calcified carotid artery atheromas on the panoramic radiographs of patients with type 2 diabetes mellitus. Oral Surg Oral Med Oral Pathol Oral Radiol Endod 89:420-424

21. Cohen SN, Friedlander AH, Jolly DA, Date L (2002) Carotid calcification on panoramic radiographs: an important marker for vascular risk. Oral Surg Oral Med Oral Pathol Oral Radiol Endod 94:510-514

22. Craven TE, Ryu JE, Espeland MA et al (1990) Evaluation of the associations between carotid artery atherosclerosis and coronary artery stenosis. A case-control study. Circulation 82:1230-1242

23. Carter LC (2000) Discrimination between calcified triticeous cartilage and calcified carotid atheroma on panoramic radiography. Oral Surg Oral Med Oral Pathol Oral Radiol Endod 90:108-110

24. Friedlander AH (1995) Panoramic radiography: the differential diagnosis of carotid artery atheromas. Spec Care Dent 15:223-227

25. Almog DM, Illig KA, Carter LC et al (2004) Diagnosis of nondental conditions. Carotid artery calcifications on panoramic radiographs identify patients at risk for stroke. N Y State Dent J $70: 20-25$ 
26. Friedlander AH, Altman L (2001) Carotid artery atheromas in postmenopausal women. Their prevalence on panoramic radiographs and their relationship to atherogenic risk factors. J Am Dent Assoc 132:1130-1136

27. Friedlander AH, Friedlander IK, Yueh R, Littner MR (1999) The prevalence of carotid atheromas seen on panoramic radiographs of patients with obstructive sleep apnea and their relation to risk factors for atherosclerosis. J Oral Maxillofac Surg 57:516-521

28. Sung EC, Friedlander AH, Kobashigawa JA (2004) The prevalence of calcified carotid atheromas on the panoramic radiographs of patients with dilated cardiomyopathy. Oral Surg Oral Med Oral Pathol Oral Radiol Endod 97:404-407

29. Madden RP, Hodges JS, Salmen CW et al (2007) Utility of panoramic radiographs in detecting cervical calcified carotid atheroma. Oral Surg Oral Med Oral Pathol Oral Radiol Endod 103:543-548

30. Tohno S, Tohno Y (1998) Age-related differences in calcium accumulation in human arteries. Cell Mol Biol 44:1253-1263

31. Elliott RJ, McGrath LT (1994) Calcification of the human thoracic aorta during aging. Calcif Tissue Int 54:268-273

32. Dunmore-Buyze PJ, Moreau M, Fenster A, Holdsworth DW (2002) In vitro investigation of calcium distribution and tissue thickness in the human thoracic aorta. Physiol Meas 23:555-566

33. Yu SY, Blumenthal HT (1963) The calcification of elastic fibers. I. Biochemical studies. J Gerontol 18:119-126

34. Sadoshima S, Kurozumi T, Tanaka K et al (1980) Cerebral and aortic atherosclerosis in Hisayama, Japan. Atherosclerosis 36:117-126

35. Aronow WS, Ahn C, Kronzon I, Gutstein H, Schoenfeld MR (1997) Association of extracranial carotid arterial disease, prior atherothrombotic brain infarction, systemic hypertension, and left ventricular hypertrophy with the incidence of new atherothrombotic brain infarction at 45-month follow-up in 1,482 older patients. Am J Cardiol 79:991-993

36. Monsour PA, Romaniuk K, Hutchings RD (1991) Soft tissue calcifications in the differential diagnosis of opacities superimposed over the mandible by dental panoramic radiography. Aust Den J 36:94-101

37. Sitzmann F (1993) When are radiographs needed in diagnostics and therapy? Dtsch Zahnarztl Z 48

38. Kopp H, Ludwig M (2007) Doppler- und Duplexsonographie. Thieme, Stuttgart, pp 1-20

39. Almog DM (2007) Utility of panoramic radiographs in detecting cervical calcified carotid atheroma. Oral Surg Oral Med Oral Pathol Oral Radiol Endod 104:451

40. Friedlander AH, Friedlander IK (1998) Identification of stroke prone patients by panoramic radiography. Aust Dent J 43:51-54

41. Farman AG, Farman TT, Khan $Z$ et al (2001) The role of the dentist in detection of carotid atherosclerosis. SADJ 56:549-553

42. Friedlander AH, Freymiller EG (2003) Detection of radiationaccelerated atherosclerosis of the carotid artery by panoramic radiography. A new opportunity for dentists. J Am Dent Assoc 134:1361-1365

43. Friedlander AH, Friedlander IK (1996) Panoramic dental radiography: an aid in detecting individuals prone to stroke. Br Dent J 181:23-26

44. Friedlander AH, Friedlander IK (1996) Identification of stroke prone patients by panoramic dental radiography. Oral Health 86 (7):9-10 